\title{
Partição de nicho por grupos funcionais de espécies arbóreas em uma floresta subtropical
}

Niche partitioning by functional groups of tree species in a subtropical forest

\author{
Karine Souza ${ }^{1}$, Pedro Higuchi ${ }^{1,2}$, Ana Carolina da Silva ${ }^{1}$, Marcos Benedito Schimalski ${ }^{1}$, Rodineli Loebens ${ }^{1}$, \\ Fernando Buzzi Júnior ${ }^{1}$, Chayane Cristina de Souza ${ }^{1}$, Luiz Carlos Rodrigues Junior ${ }^{1}$, Felipe Fornara Walter ${ }^{1}$, \\ Francieli de Fátima Missio ${ }^{1}$ \& Angélica Dalla Rosa ${ }^{1}$
}

\begin{abstract}
Resumo
O presente estudo teve como objetivo investigar a variação de atributos funcionais de espécies arbóreas ao longo de diferentes posições topográficas, em uma floresta subtropical na região do Alto Uruguai, em Santa Catarina. Para as 20 espécies mais abundantes na área amostrada, foram determinados densidade básica da madeira (WD), altura potencial (Hmax), área foliar (LA), área foliar específica (SLA), regime de renovação foliar, guildas de regeneração e de dispersão. A estrutura funcional foi avaliada por meio dos valores dos atributos funcionais por parcela, ponderados para a comunidade (CWM). A partir de um Modelo Digital do Terreno na resolução de 1 $\mathrm{m}$, foram extraídas para cada parcela a elevação, a declividade e curvatura. Os dados foram analisados por meio de correlações lineares, Análise de Coordenadas Principais (PCoA), Análises de Componente Principais (PCA) e modelo linear simples. Os resultados demonstraram correlações significativas e negativas entre Hmax x LA e Hmax x SLA. O gradiente topográfico influenciou de forma significativa a estrutura funcional do componente arbóreo. Conclui-se que a ocupação de diferentes posições no gradiente topográfico e ao longo do perfil vertical da floresta se deve ao fato das espécies apresentarem diferentes estratégias ecológicas.
\end{abstract}

Palavras-chave: atributos funcionais, ecologia florestal, ecologia funcional, mata atlântica, topografia.

\begin{abstract}
This study aimed to investigate functional attributes of tree species along different topographic position, in a forest located in Upper Uruguay region, in Santa Catarina. The wood density (WD), potential height (Hmax), leaf area (LA), specific leaf area (SLA), leaves renovation regime, regeneration and dispersal guilds were determined for the 20 most abundant species in the sampled area. The functional structure was evaluated through a community weight matrix (CWM) of traits values for each sampling unit. Mean values of elevation, declivity and curvature were extracted for each sampling unit, from a Terrain Digital Model in a resolution of $1 \mathrm{~m}$. The data was analyzed through linear correlations, Principal Coordinates Analysis (PCoA), Principal Components Analysis (PCA) and linear simple model. The results indicated significant and negative correlation between Hmax x LA and Hmax x SLA. The topographic gradient significantly influenced the functional structure of tree component. It is concluded that the occupation of different positions along the topographic gradient and the forest vertical profile by tree species were mediated by different ecological strategies.
\end{abstract}

Key words: functional attributes, forest ecology, functional structure, Atlantic forest, topography.

\section{Introdução}

Recentemente, estudos com uma abordagem funcional têm contribuído para uma melhor compreensão sobre os padrões de coexistência e segregação de espécies arbóreas em comunidades
(Adler et al. 2013) e sobre as respostas das espécies em função de variações no ambiente (Rosado et al. 2016). Abordagens com esta natureza baseiam-se no pressuposto de que a capacidade de desenvolvimento de uma espécie

\footnotetext{
'Universidade do Estado de Santa Catarina, Depto. Engenharia Florestal, Av. Luiz de Camões 2090, 88520-000, Lages, SC, Brasil.

${ }^{2}$ Autor para correspondência: higuchip@gmail.com
} 
em um determinado habitat está relacionada com sua estratégia de uso dos recursos, que pode ser sintetizada a partir de seus traços funcionais ("functional traits"). Segundo Violle et al. (2007), os traços funcionais de plantas podem ser definidos como sendo as características morfológicas, fisiológicas e fenológicas que impactam diretamente a performance dos indivíduos (crescimento, reprodução e sobrevivência), influenciando, assim, na aptidão dos mesmos a uma determinada condição ambiental. Assim, investigações sobre as relações entre os traços funcionais e a heterogenidade ambiental permitem um maior entendimento sobre o estabecimento das espécies em um determinando ambiente (McGill et al. 2006), sobre os mecanismos de coexistência de espécies arbóreas (Silvertown et al. 1999), sobre a partição de nichos ecológicos (Ackerly \& Cornwell 2007; Violle \& Jiang 2009) e do funcionamento do ecossistema (Díaz \& Cabido 2001). Ressalta-se que a ideia da relação entre as caracteristicas funcionais e o ambiente não é nova, sendo que, no século XIX, Darwin relatou que o processo de radiação evolutiva, em diferentes grupos de plantas e animais, pode produzir variações fenotípicas associadas às caracteristicas ambientais (Losos \& Ricklefs 2009). Ainda, em meados do século passado, MacArthur \& Levin (1967) introduziram o termo "similaridade limitante", demonstrando que a especialização das espécies está relacionada com a heterogenidade ambiental e que a competição inter-específica pode impor um limite para a similiraridade funcional de espécies co-existentes.

A partir de seus atributos, que representam os diferentes valores que um determinado traço funcional podem assumir (Violle et al. 2007), alguns autores (Díaz et al. 2004; Reich 2014) têm agrupado as espécies nas seguintes categorias relacionadas às suas estratégias ecológicas: i) conservadoras que possuem atributos que refletem uma elevada capacidade de proteção da biomassa (e.g., elevada densidade da madeira, folhas pequenas, baixa área foliar específica); e ii) aquisitivas - que apresentam atributos que refletem uma maior capacidade de maximizar a obtenção de recursos (e.g., baixa densidade da madeira, folhas maiores e maior área foliar específica). De forma geral, enquanto espécies com estratégias conservadoras são favorecidas em ambientes com menor disponibilidade de recursos (e.g., solos pobres, baixa disponbilidade lúminica, com déficit hidrico), as que apresentam estratégias aquisitivas são favorecidas em ambientes com maior disponibilidade de recursos (e.g., solos de maior fertilidade, maior disponibilidade luminínica, sem defícit hídrico).

Destaca-se que a topografia tem sido frequentemente relatada como uma importante fonte de heterogeneidade de comunidades de espécies arbóreas em escala local (e.g., Oliveira Filho et al. 1994; Valencia et al. 2004; Yang et al. 2014). Isto ocorre, pois, ao longo de um gradiente topográfico, observa-se variações ambientais associadas ao tipo de solo, à declividade, à umidade (Oliveira Filho et al. 1998) e ao microclima (Ackerly et al. 2002), de forma que áreas de baixada geralmente apresentam características mais favoráveis para o desenvolvimento de plantas, como, por exemplo, maior umidade e fertilidade do solo e menor demanda evapotranspirativa, do que áreas de encosta e topo. Desta forma, esperase que a distribuição de espécies florestais, em diferentes posições do relevo, seja mediada por seus respectivos atributos funcionais, uma vez que os mesmos respondem a diferentes condições ambientais (Körner 2003; Ackerly 2004).

Considerando a importância de estudos ecológicos com uma abordagem funcional para o entendimento da organização de comunidades biológicas, o presente trabalho teve como principal objetivo investigar as variações dos atributos funcionais de espécies arbóreas ao longo de diferentes hábitats, em posições topográficas distintas, em uma floresta subtropical no Sul do Brasil. Nesta região, as florestas foram reduzidas e estão sob constante pressão antrópica, o que justifica estudos com abordagens ecológicas, que podem contribuir para subsidiar a restauração e uso sustentável. Assim, testou-se a hipótese de que o ambiente de baixada topográfica possa favorecer a predominância de estratégias aquisitivas e a encosta favorece estratégias conservadoras.

\section{Material e Métodos}

Área de estudo

O estudo foi realizado em uma área ecotonal entre a Floresta Ombrófila Mista e a Floresta Estacional Decidual (IBGE 2012), em um fragmento florestal em avançado estágio de sucessão. A área localiza-se no município de Capão Alto, SC (coordenadas UTM: 524407 L, $6881056 \mathrm{~N}$; altitude aproximada de $700 \mathrm{~m}$ ), junto ao Reservatório UHE Barra Grande, do rio Pelotas, na Bacia Hidrográfica do Rio Uruguai, que foi alagado no ano de 2005. O clima, de acordo com Köppen, é do tipo Cfb. A precipitação média anual varia entre 1.200 a 1.900 $\mathrm{mm}$, com chuvas bem distribuídas durante o ano, e a 
temperatura média anual é de $16^{\circ} \mathrm{C}$ (Formento et al. 2004). Os solos variam de Cambissolo a Neossolo (Souza et al. 2015).

\section{Amostragem da vegetação}

Os valores de atributos funcionais [densidade básica da madeira (WD) $\left(\mathrm{g} / \mathrm{cm}^{3}\right)$, área foliar (LA) $\left(\mathrm{cm}^{2}\right)$, área foliar específica (SLA) $\left(\mathrm{cm}^{2} / \mathrm{g}\right)$, altura potencial (Hmax) (m), regime de renovação foliar, guildas de dispersão] e a performance ecológica quanto à disponibilidade de luz (guildas de regeneração) foram determinados para as 20 espécies mais abundantes amostradas por Souza et al. (2015) na mesma área. Souza et al. (2015) amostraram o componente arbóreo (DAP maior ou igual a $5 \mathrm{~cm}$ ) utilizando 50 parcelas de $10 \times$ $20 \mathrm{~m}$, distribuídas de forma sistemática, com uma distância de $5 \mathrm{~m}$ entre si, em uma grade de $5 \times$ 10 parcelas, com o maior lado acompanhando a encosta no sentido de maior declividade. Desta forma, totalizou-se 1 ha de amostragem, em uma área florestal contínua de, aproximadamente, 250 ha. As espécies avaliadas no presente estudo compuseram $93,15 \%$ dos indivíduos lenhosos e $89,15 \%$ da área basal amostrada, sendo, portanto, representativas da estrutura da área amostrada da floresta.

Para a determinação da densidade básica da madeira, utilizou-se o protocolo de Chave (2006), coletando, assim, uma amostra de madeira de cinco a 10 indivíduos por espécie, com diâmetro na altura do peito de 10 a $30 \mathrm{~cm}$, por meio de um trado de incremento (diâmetro de $5,15 \mathrm{~mm}$ ). As amostras foram acondicionadas em recipientes com água até que apresentassem massa úmida constante. $\mathrm{O}$ peso da massa úmida constante foi determinado pelo princípio de Arquimedes, em que a medida da massa de água deslocada é igual a massa úmida da amostra. Posteriormente, as amostras foram colocadas em estufa até que a massa seca permanecesse constante e então pesadas em balança analítica com precisão de 0,001 g. Dessa maneira, a razão entre massa seca $(\mathrm{g})$ pela massa úmida (g) gerou a densidade básica da madeira. A área foliar e a área foliar específica foram obtidas por meio da coleta de 20 folhas por indivíduo (10 indivíduos/espécie), incluindo os pecíolos e, para folhas compostas, raques, seguindo o protocolo de Pérez-Harguindeguy et al. (2013). As folhas foram fotografadas por meio de uma câmera acoplada a uma estrutura de madeira com altura fixa. A imagem da folha foi então classificada no programa Envi e foi obtida a área da folha no programa ArcGis. Em seguida, as folhas foram armazenadas em estufa até alcançarem a massa seca constante e pesadas em balança analítica com precisão de 0,001 g. A área foliar específica foi obtida pela a razão entre a área foliar $\left(\mathrm{cm}^{2}\right)$ e a massa seca das folhas $(\mathrm{g})$. Os atributos funcionais referentes à altura máxima potencial, regime de renovação foliar (perenes, semidecíduas e decíduas) e guildas de dispersão (zoocóricas, anemocóricas e autocóricas, conforme Van der Pijl 1982); e a performance ecológica quanto à disponibilidade de luz (climácicas exigentes em luz e climácicas tolerantes à sombra, conforme Oliveira Filho et al. 1994, adaptado de Swaine \& Whitmore 1988) foram caracterizados por meio de observações no campo e consulta à literatura (e.g., Leyser et al. 2012; Negrini et al. 2012; Silva et al. 2012).

\section{Topografia}

A caracterização topográfica da área foi realizada a partir das coordenadas geográfica das parcelas, obtidas com um GPS de navegação, e de um modelo digital do terreno (MDT), derivado de imagens aéreas obtidas pelo recobrimento aéreo estadual de Santa Catarina, na resolução de $1 \mathrm{~m}$. A partir do MDF, derivou-se, por meio do programa ArcGis, junto com a extensão 3D Analyst, os valores médios para cada parcela de elevação, representando a altitude $(\mathrm{m})$, declividade $(\%)$ e curvatura.

\section{Análises dos dados}

Foi verificada a relação entre os atributos funcionais das espécies por meio de análises de correlações. A similaridade funcional das 20 espécies estudadas foi analisada por meio da ordenação produzida por uma Análise de Coordenadas Principais (PCoA), a partir de uma matriz de dissimilaridade funcional elaborada usando a distância de Gower. Em seguida, os traços funcionais foram ajustados à ordenação produzida por meio da função envfit, do pacote Vegan (Oksanen et al. 2016), sendo aqueles significativos $(p<0,001)$ plotados na forma de vetores. Os valores para as parcelas referentes aos atributos funcionais ponderados para a comunidade (CWM) e às variáveis topográficas foram ordenados por meio de duas Análises de Componentes Principais (PCA's). Com o propósito de identificar os eixos das PCA's que sintetizaram de forma significativas os gradientes funcionais e topográficos, foram realizadas análises de Scree Plot (Jackson 1993). A magnitude dos scores das parcelas para os eixos 
das PCA's, que explicaram proporção significativa da variação total dos dados, foram espacializados e apresentados na forma de mapas. A fim de se avaliar a influência do gradiente topográfico sobre os atributos funcionais da comunidade, utilizou-se modelos lineares simples, tendo como variáveis independentes os eixos da PCA topográfica, que explicaram proporções significativas da inércia total; e como variáveis dependentes, os eixos da PCA funcional, que explicaram proporções significativas da inércia total. Todas as análises foram realizadas no programa $\mathrm{R}$ ( $\mathrm{R}$ Core Team 2016), junto com os pacotes FD (Laliberté \&
Legendre 2010; Laliberté \& Shipley 2011) e Vegan (Oksanen et al. 2016).

\section{Resultados}

Os resultados demonstram que as espécies avaliadas apresentaram variações em seus atributos funcionais e em exigência lumínica (Tab. 1 e Fig. 1), o que sugere a existência de diferentes estratégias de vida entre as mesmas. A maior parte das espécies foi classificada como semidecídua, zoocórica e clímax exigentes em luz. Os valores médios dos atributos funcionais avaliados foram de 0,5940 g. $\mathrm{cm}^{-3}\left(0,3460\right.$ a 0,7968 g.cm $\left.\mathrm{cm}^{-3}\right)$ para densidade

Tabela 1 - Atributos funcionais referentes à densidade básica da madeira (WD, g.cm $\left.{ }^{-3}\right)$, área foliar $\left(\mathrm{LA}, \mathrm{cm}^{2}\right)$, área foliar específica (SLA, $\mathrm{cm}^{2} \cdot \mathrm{g}^{-1}$ ), altura potencial (Hmax, $\mathrm{m}$ ), regime de renovação foliar (Dec), guildas de dispersão (Gdis) e guildas de regeneração (Greg) das 20 espécies arbóreas mais abundantes amostradas, em uma floresta subtropical em Santa Catarina.

Table 1 - Functional attributes related to wood density $\left(\mathrm{WD}, \mathrm{g} \cdot \mathrm{cm}^{-3}\right)$, leaf area $\left(\mathrm{LA}, \mathrm{cm}^{2}\right)$, specific leaf area $\left(\mathrm{SLA}, \mathrm{cm}^{2} \cdot \mathrm{g}^{-1}\right)$, maximum potential height (Hmax, m), leaves renovation regime (Dec), dispersal guilds (Gdis) and regeneration guilds (Greg) for the 20 most abundant sampled species, in a subtropical forest in Santa Catarina.

\begin{tabular}{lccccccc}
\hline Espécies & WD & LA & SLA & Hmax & Dec & Gdis & Greg \\
\hline Allophylus edulis (A.St.-Hil., Cambess. \& A. Juss.) Radlk. & 0,5259 & 27,95 & 200,90 & 20 & SD & Zoo & CEL \\
Annona rugulosa (Schltdl.) H. Rainer & 0,3460 & 28,59 & 246,74 & 15 & D & Zoo & CEL \\
Banara tomentosa Clos & 0,5569 & 20,42 & 312,90 & 14 & SD & Zoo & CTS \\
Campomanesia xanthocarpa O.Berg & 0,6761 & 27,69 & 198,47 & 20 & D & Zoo & CEL \\
Casearia decandra Jacq. & 0,6345 & 8,67 & 266,41 & 12 & D & Zoo & CTS \\
Cupania vernalis Cambess. & 0,5696 & 382,97 & 103,65 & 25 & SD & Zoo & CEL \\
Eugenia uniflora L. & 0,7968 & 5,71 & 280,78 & 20 & SD & Zoo & CEL \\
Lonchocarpus campestris Mart. ex Benth. & 0,6041 & 47,89 & 238,95 & 12 & D & Zoo & CEL \\
Luehea divaricata Mart. \& Zucc. & 0,5172 & 52,21 & 243,78 & 27 & D & Ane & CEL \\
Machaerium paraguariense Hassl. & 0,5591 & 78,82 & 241,31 & 20 & SD & Ane & CEL \\
Matayba elaeagnoides Radlk. & 0,4679 & 169,64 & 123,57 & 25 & SD & Zoo & CEL \\
Myrcianthes gigantea (D. Legrand) D. Legrand & 0,7531 & 11,23 & 82,70 & 26 & SD & Zoo & CEL \\
Myrcianthes pungens (O.Berg) D. Legrand & 0,6578 & 10,02 & 82,49 & 25 & SD & Zoo & CEL \\
Myrocarpus frondosus Allemão & 0,7223 & 91,05 & 199,24 & 30 & D & Ane & CEL \\
Nectandra megapotamica (Spreng.) Mez & 0,4095 & 18,41 & 161,02 & 28 & P & Zoo & CEL \\
Ocotea puberula (Rich.) Nees & 0,4401 & 31,79 & 146,53 & 26 & SD & Zoo & CEL \\
Parapiptadenia rigida (Benth.) Brenan & 0,6830 & 76,15 & 129,51 & 25 & D & Aut & CEL \\
Pilocarpus pennatifolius Lem. & 0,7491 & 168,44 & 94,20 & 13 & SD & Aut & CTS \\
Sebastiania brasiliensis Spreng. & 0,6137 & 10,31 & 179,50 & 6 & SD & Aut & CTS \\
Sebastiania commersoniana (Baill.) L.B. Sm. \& Downs & 0,5969 & 9,91 & 152,18 & 21 & D & Aut & CEL \\
\hline Média & 0,5940 & 63,89 & 184,24 & 20,50 & - & - & - \\
\hline
\end{tabular}


básica da madeira (WD); $63,89 \mathrm{~cm}^{2}(5,71$ a 382,97 $\left.\mathrm{cm}^{2}\right)$ para a área foliar (LA); $184,24 \mathrm{~cm}^{2} \cdot \mathrm{g}^{-1}(82,49$ a $\left.312,90 \mathrm{~cm}^{2} \cdot \mathrm{g}^{-1}\right)$ para área foliar específica (SLA) e $20,50 \mathrm{~m}$ (6 a $30 \mathrm{~m})$ para altura potencial (Hmax).

As espécies com o maior e menor valores de WD foram, respectivamente, Pilocarpus pennatifolius Lem. (0,7491 g.cm $\left.{ }^{-3}\right)$ (autocórica, clímax tolerante ao sombreamento e semidecídua) e Annona rugulosa (Schltdl.) H. Rainer (0,3460 g.cm ${ }^{-3}$ ) (zoocórica, clímax exigente em luz e decídua). Enquanto Cupania vernalis Cambess. foi a espécie com as folhas de maior tamanho, Eugenia uniflora L. apresentou as menores folhas. Em relação à SLA, atributo relacionado a dureza das folhas, Banara tomentosa Clos e E. uniflora apresentaram os maiores valores, ou seja, folhas mais membranáceas. Ao contrário, Myrcianthes gigantea (D. Legrand) D. Legrand e Myrcianthes

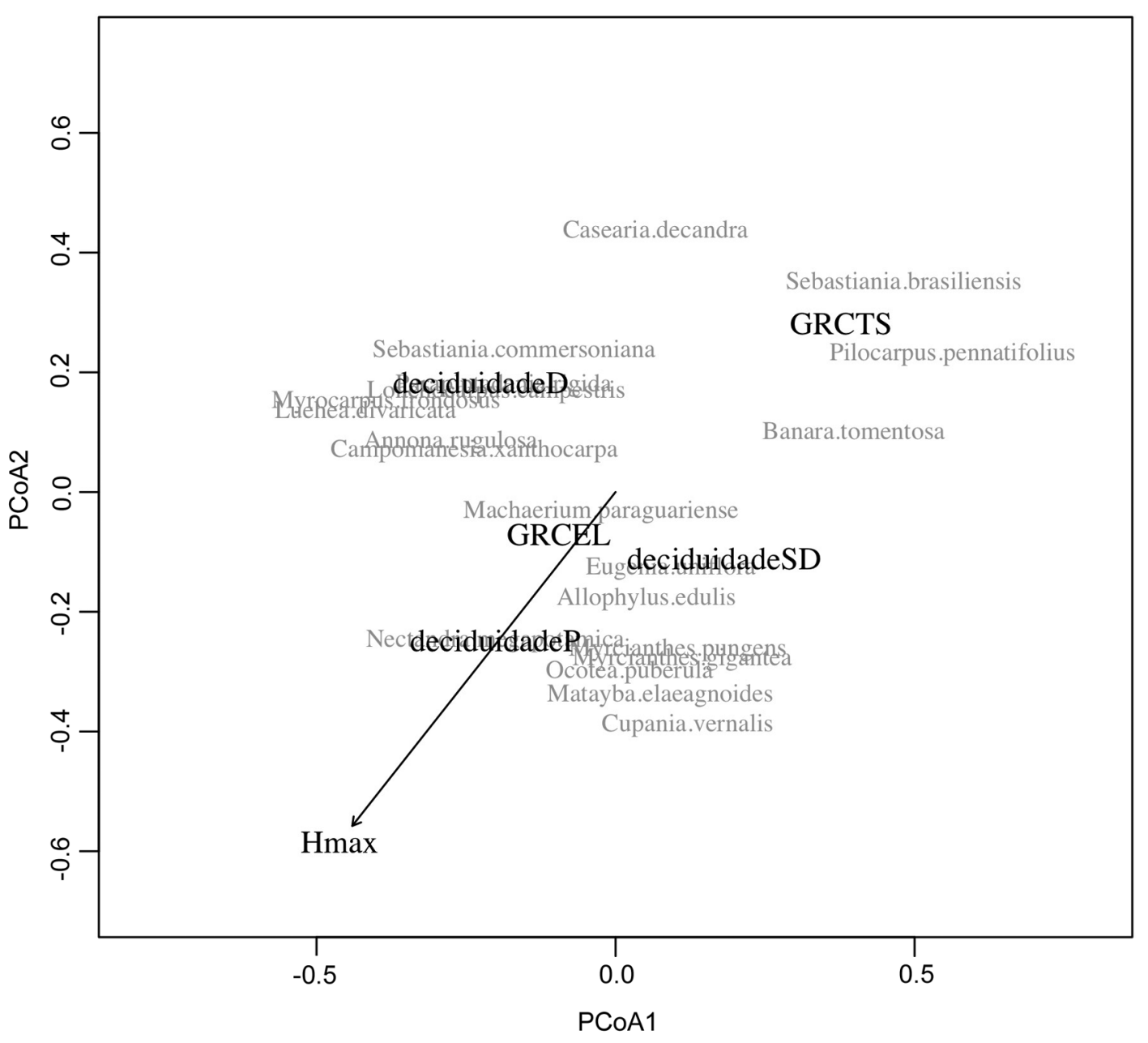

Figura 1 - Ordenação dos atributos funcionais, por meio da Análise de Coordenadas Principais (PCoA), das 20 espécies mais abundantes amostradas em uma floresta subtropical em Santa Catarina. Traços funcionais (deciduidadeD = Decídua; deciduidadeSD = semi-decídua; deciduidadeP = perene; Hmax = Altura potencial) e performance ecológica quanto à disponibilidade de luz (GRCTS = clímax tolerante ao sombreamento; GRCEL = clímax exigente em luz) com relação significativa $(\mathrm{p}<0,001)$ com a ordenação produzida.

Figure 1 - Functional attributes ordination, by Principal Coordinates Analysis (PCoA), for the 20 most abundant species sampled in a subtropical forest in Santa Catarina. Functional traits (deciduidadeD $=$ deciduous; deciduidadeSD $=$ semi-deciduous; deciduidadeP = perennial; Hmax = Potential height) and light requirement ecological performance $($ GRCTS $=$ shade tolerant climax; GRCEL = light demanding climax $)$ with significant $(\mathrm{p}<0.001)$ relation to produced ordination. 
pungens (O.Berg) D. Legrand apresentaram folhas mais coriáceas. As espécies com as maiores alturas potenciais foram Myrocarpus frondosus Allemão (30 m) e Nectandra megapotamica (Spreng.) Mez (28 m), ambas classificadas como clímax exigente em luz, e as com menores alturas potenciais foram Sebastiania brasiliensis Spreng. (6 m), Casearia decandra Jacq. (12 m), classificadas como clímax tolerante à sombra, e Lonchocarpus campestris Mart. ex Benth. (12 m), clímax exigente em luz. Enquanto as espécies com os maiores valores de altura potencial, classificadas como clímax exigentes em luz, são aquelas que podem alcançar o dossel da floresta, as espécies com os menores valores de altura potencial são, predominantemente, clímax tolerantes à sombra, que representam aquelas restritas ao sub-bosque.

A ordenação das espécies em função de seus atributos funcionais (Fig. 1) indicou variações associadas, principalmente, à exigência lumínica, ao regime de renovação foliar e altura potencial. Os eixos 1 e 2 da Análise de Coordenadas Principais (PCoA) apresentaram auto-valores de, respectivamente, 1,37 e 1,17, que explicaram, juntos, $43,01 \%$ da inércia total $(5,89)$. O Eixo 1 separou as espécies exigentes em luz e de maior porte, ordenadas mais a esquerda, das tolerantes ao sombreamento e de menor porte, ordenadas mais a direita. Pode-se observar que as espécies exigentes em luz, predominantemente a esquerda da ordenação, distribuiram-se ao longo do Eixo 2, com as decíduas ocupando a parte superior da ordenacão, e as perenes/semidecíduas ordenadas na parte inferior.

No que se refere à relação entre os atributos funcionais de valores contínuos das espécies (Fig. 2), observaram-se correlações marginalmente significativas entre SLA e LA e entre SLA e Hmax. Ambas correlações foram negativas, ou seja, quanto maior SLA, que indica folhas membranáceas, menores os valores de tamanho de folhas e de altura potencial.

De acordo com a análise do gráfico de Scree plot (Fig. 3a), referente a ordenação dos valores de atributos funcionais das parcelas, ponderados para a comunidade (CWM), apenas o Eixo 1 explicou uma proporção significativa da inércia $(2,87)$, que representa $71,67 \%$ da variação total dos dados. O Eixo 1 da PCA apresentou correlação positiva com valores de WD e SLA e correlação negativa com LA e Hmax (Fig. 3b). Assim, é possível identificar um gradiente funcional na área, com um extremo, ordenado a esquerda, representado por parcelas com árvores de maior porte, maior LA, baixa WD e baixa SLA; e outro extremo, ordenado a direita, por parcelas com árvores de pequeno porte, folhas menores, maior WD e maior SLA.

Em relação à ordenação das variáveis topográficas, a análise do gráfico Scree Plot também indicou que apenas o Eixo 1 explicou uma parte significativa da variação total $(70,35 \%)$ (Fig. 3c). Assim, pode-se inferir que as parcelas se distribuíram em função de um gradiente topográfico sintetizado ao longo do Eixo 1 da PCA, com os valores de altitude, declividade e curvatura, variando de, respectivamente, 646 a $710 \mathrm{~m}, 23$ a $49 \%$ e $-0,34$ a 2,51. Quanto ao valor de curvatura, valores negativos indicam curvatura côncava e valores positivos indicam parcelas convexas. Assim, ordenadas no lado esquerdo da PCA encontram-se as parcelas com menor altitude (alt) e com menores valores de curvatura (curv) e de declividade (decl) (Fig. 3d), que são aquelas situadas na baixada próxima da represa. No lado direito da ordenação, estão situadas as parcelas com maior altitude, com curvatura predominantemente convexa e maior declividade, que são aquelas situadas ao longo da encosta.

A espacialização dos valores dos Eixos 1 das PCA's topográfica (Fig. 4a) e funcional (Fig. 4b) demonstra a existência de sobreposição espacial, indicando correlação entre estes valores. De fato,

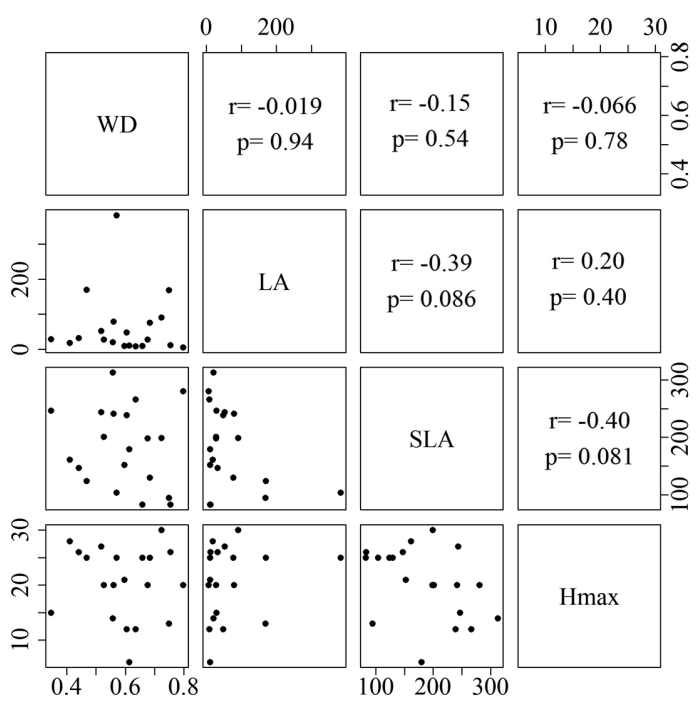

Figura 2 - Correlações entre os atributos funcionais das espécies avaliadas, em uma floresta subtropical em Santa Catarina.

Figure 2 - Correlations among functional attributes of evaluated species, in a subtropical forest in Santa Catarina. 
a regressão linear entre estas variáveis indicou correlação positiva significativa $\left(\mathrm{R}^{2}=0,5276, p\right.$ $<0,01)$, demonstrando que parcelas com maiores valores no Eixo 1 da PCA topográfica também apresentam maiores valores no Eixo da PCA funcional. Este padrão indica que a área de encosta, de maior elevação, terreno predominantemente convexo e com maior declividade, apresenta o componente arbóreo caracterizado por apresentar árvores de menor porte, maior WD, menor LA e maior SLA. Já nos locais de baixada, com menor elevação e plano, o componente arbóreo foi
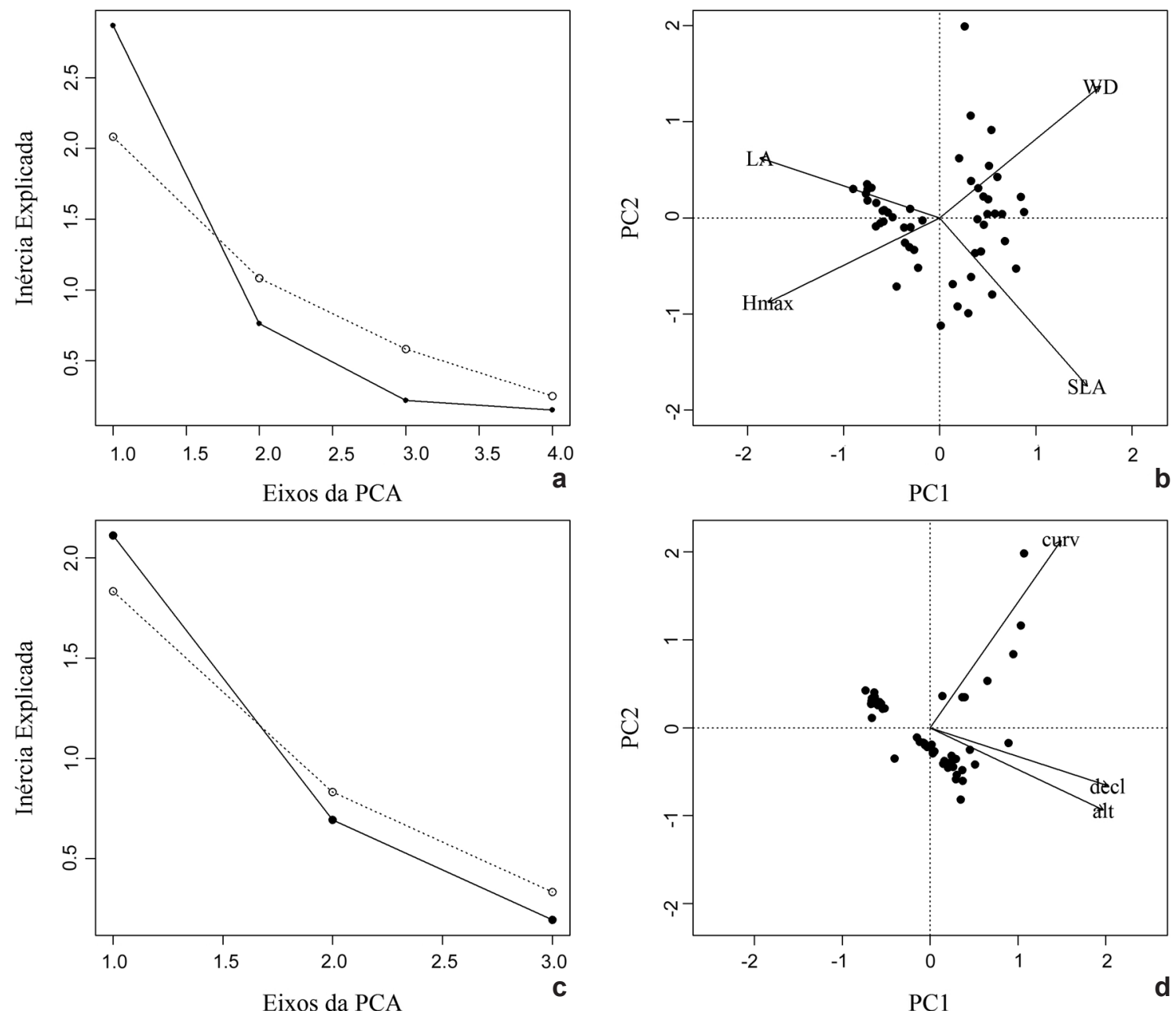

Figura 3 - Scree plots (a,c.) para os resultados das ordenações produzidas a partir das Análises de Componentes Principais dos atributos funcionais, ponderados para a comunidade (CWM) (b.) e variáveis topográficas (d.), para cada parcela, em uma floresta subtropical em Santa Catarina. Em (a.) e (c.), a linha contínua representa os valores de inércia explicado por cada eixo da PCA e a linha pontilhada indica valores aleatórios de acordo com a distribuição de Broken Stick. Se a linha contínua encontra-se acima da linha pontilhada, a proporção da variação explicada pelo eixo da PCA é significativa. (b. LA = área foliar; Hmax = altura potencial; WD = densidade da madeira; SLA = área foliar específica. $d$. curv = curvatura; decl = declividade; alt = altitude).

Figure 3 - Scree plots (a,c.) for the results of ordinations produced by Principal Component Analysis of community weight means (CWM) functional attributes (b.) and topographic variables (d.) for each sampling units, in a subtropical forest in Santa Catarina. In (a.) and (c.), the solid line represents the inertia values explained by each PCA axis and dotted line indicates random values according to Broken Stick distribution. If the solid line is above the dotted line, the proportion of variation explained by PCA axis is significant. (b. LA = leaf area; Hmax = potential height; $\mathrm{WD}=$ wood density; SLA = specific leaf area. $\mathrm{d}$. curv = curvature; decl $=$ declivity; alt $=$ elevation). 

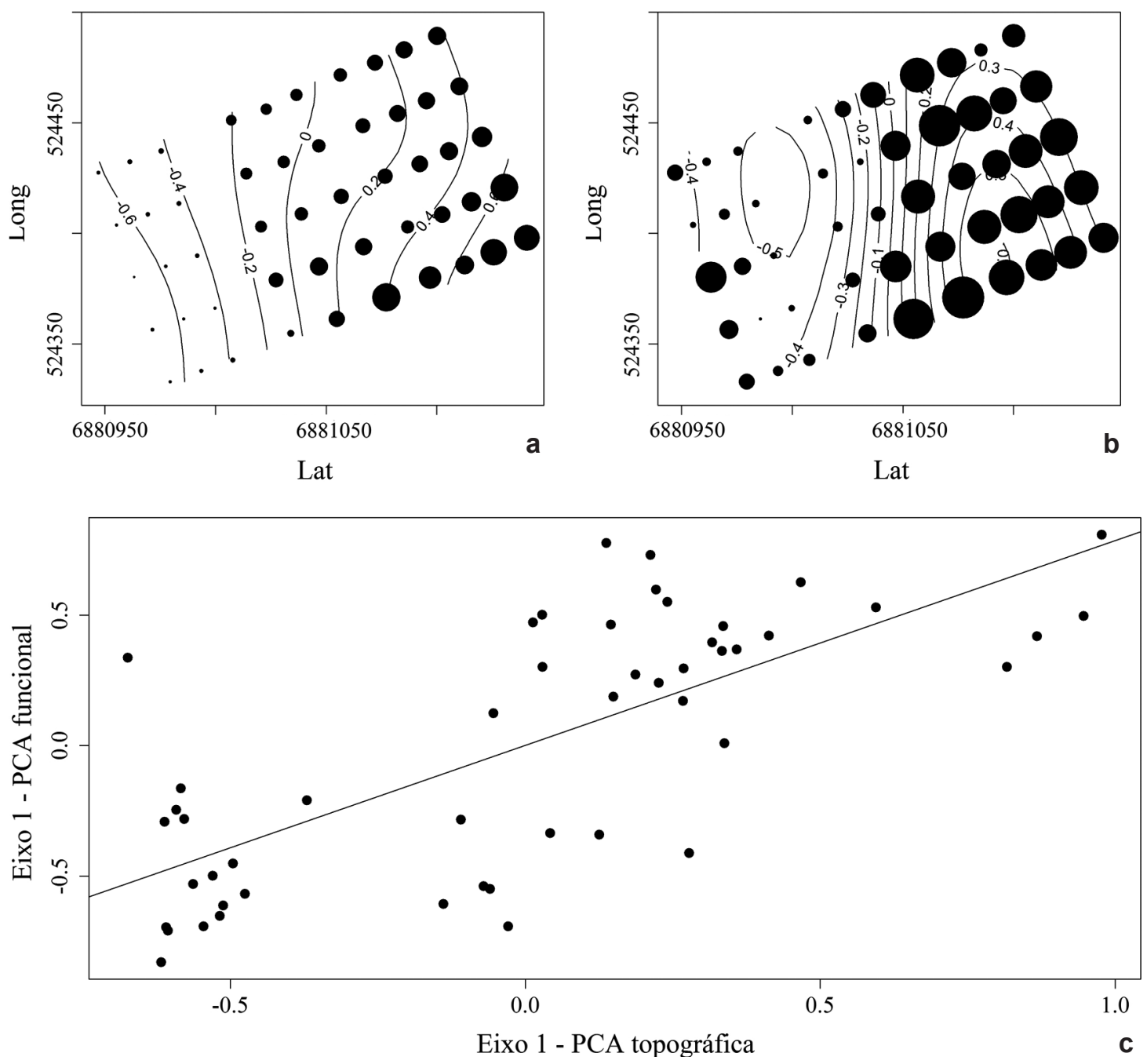

Figura 4 - Espacialização dos eixos 1 da PCA topográfica (a.) e da PCA funcional (b.); e regressão linear simples (c.) entre estas variáveis, em uma floresta subtropical em Santa Catarina.

Figure 4 - Spatialization of axis 1 of topographic (a.) and functional PCA (b.); and simple linear regression (c.) between both variables, in a subtropical forest in Santa Catarina.

caracterizado por apresentar árvores de maior porte, menor WD, maior LA e menor SLA.

\section{Discussão}

Em síntese, nossos resultados demonstraram i) que as espécies avaliadas apresentaram variações em seus respectivos atributos funcionais, principalmente no que se refere ao regime de renovação foliar e altura potencial, e performance ecológica quanto à disponibilidade de luz; ii) correlações marginalmente significativas e negativas entre área foliar específica (SLA) e área foliar (LA) e entre SLA e altura potencial
(Hmax); e iii) variação em escala local dos atributos funcionais em função do relevo, sendo que, enquanto na encosta predominaram árvores de menor porte, com maiores valores de WD e de SLA, e menores valores LA; na baixada observouse o oposto, com a predominância de árvores maior porte, com menores valores de WD e SLA, e maiores valores de LA.

As estratégias ecológicas de espécies arbóreas para a alocação de biomassa têm sido categorizadas em dois tipos: estratégias conservadoras, que permitem a conservação e retenção de recursos obtidos a partir de tecidos protegidos (e.g., elevada 
densidade da madeira) ou estratégias aquisitivas, que permitem a rápida obtenção de recursos (e.g., baixa densidade da madeira) (Carreño-Rocabado et al. 2012). Assim, de forma geral, os resultados evidenciaram as diferentes estratégias que as espécies apresentaram para a partição de nichos ecológicos, tanto para ocuparem os diferentes setores topográficos, quanto para se distribuírem ao longo do perfil vertical da floresta.

Enquanto as espécies que se desenvolvem no sub-bosque apresentam a luz como um recurso limitante, as que ocupam o dossel têm acesso a plena luz solar, além de estarem sujeitas a um ambiente com maior exposição ao vento e abrasão física provocada pelas condições microclimáticas (Thomas \& Winner 2002). A maior SLA em espécies com menor Hmax, indicando folhas mais membranáceas, tem sido relatada com uma estratégia para maximizar a captura de luz em condições de sombreamento, como no subbosque (Evans \& Poorter 2001). Já uma menor SLA em espécies como maior Hmax pode ser interpretada como uma estratégia para minimizar os danos mecânicos causados pelo microclima no dossel (Thomas \& Winner 2002), uma vez que nestas condições, além da maior disponibilidade luz, espera-se maior incidência de vento e maior amplitude diária e anual na temperatura e umidade. Da mesma forma, a Hmax também já foi identificada como um atributo funcional relacionado às estratégias de captura de luz (Weiher et al. 1999; Poorter et al. 2005), de forma que, em uma floresta mais tardia, com dossel contínuo, as espécies com menor altura potencial, como, por exemplo, Sebastiania brasiliensis, são aquelas aptas a completarem todo o ciclo de vida em uma condição de sombreamento.

A área de baixada, com menor elevação e terreno mais plano, apresentou o componente arbóreo caracterizado por árvores mais altas, com menores valores de densidade da madeira, folhas maiores e mais coriáceas. Em contrapartida, nas partes mais altas do relevo, com maior declividade, observou-se árvores mais baixas, com maior densidade da madeira e com folhas menores e mais membranáceas. Assim, confirma-se a hipótese do trabalho, pois os resultados demonstram que os atributos funcionais das espécies não ocorreram de forma homogênea no espaço, apresentando variações associadas ao gradiente topográfico existente, com o ambiente de baixada favorecendo, predominantemente, estratégias aquisitivas e, o de encosta, predominantemente, estratégias conservadoras. Isto reforça a ideia de que variações topográficas representam um dos principais fatores ecológicos promotores de heterogeneidade vegetacional em escala local (Valencia et al. 2004; Yang et al. 2014).

Considerando que WD apresenta relação inversa com a taxa de crescimento (Chave et al. 2009), é possível inferir que uma menor WD, maior Hmax e maior LA observadas na baixada estejam relacionadas com a predominância de espécies com estratégias aquisitivas, que investem em crescimento mais rápido, de forma a maximizar a aquisição de recursos, padrão tipicamente observado em ambientes com maior disponibilidade de nutrientes (Baker et al. 2003). Já maior WD, menor Hmax e menor LA observadas nos setores topográficos superiores, mais declivosos, sugerem a predominância de espécies com o crescimento mais lento, o que representa uma estratégia conservadora e de proteção contra a perda de biomassa, típico de ambientes com maior limitação de recursos (Baker et al. 2003).

Em relação à área foliar específica (SLA), estudos têm demonstrado que a ocorrência de um menor valor deste atributo é esperada em ambientes com maior incidência de radiação solar (Ackerly et al.2002) e com menor disponibilidade de água no solo (Cornwell \& Ackerly 2009). Considerando que estas condições são esperadas em posições mais elevadas da encosta (Cornwell \& Ackerly 2009), seria esperada a observação de maior SLA na baixada e menor SLA nas áreas mais altas do relevo, o que não foi observado, sendo encontrado um padrão oposto. A menor SLA na área mais baixa sugere que variações na área foliar específica estejam relacionadas com outros aspectos ecológicos de maior relevância e, ou, que o gradiente microclimático e de disponibilidade de água no solo seja curto para resultar no padrão documentado na literatura. Concluiu-se que, na floresta estudada, o relevo representou um relevante componente determinístico na organização de espécies arbóreas em pequena escala espacial. Enquanto na área da baixada predominou a ocorrência de estratégias aquisitivas, sintetizadas pelo maior porte das árvores, menor densidade da madeira e folhas de maior tamanho, na área de encosta predominou estratégias conservadoras, sintetizadas pelo menor porte das árvores, maior densidade da madeira e folhas de menor tamanho. No que se refere à aplicação ecológica dos resultados encontrados, o presente trabalho sugere que a categorização das espécies como de estratégias aquisistivas ou 
conservadoras tem grande potencial para subsidiar a seleção de espécies arbóreas em programas de restauração florestal, com o propósito de serem introduzidas em diferentes condições ambientais.

\section{Agradecimentos}

À FAPESC (Fundação de Amparo à Pesquisa e Inovação do Estado de Santa Catarina), a concessão de bolsa de Mestrado à primeira autora do trabalho. Ao CNPq, a concessão de bolsa de Produtividade para o segundo (456060/2014-6) e o terceiro (309461/2014-6) autores.

\section{Referências}

Ackerly DD (2004) Adaptation, niche conservatism, and convergence: comparative studies of leaf evolution in the California chaparral. The American Naturalist 163: 654-671.

Ackerly DD, Knight CA, Barton K \& Starmer KP (2002) Leaf size, specific leaf area and microhabitat distribution of chaparral woody plants: contrasting patterns in species level and community level analyses. Oecologia 130: 449-457.

Ackerly DD \& Cornwell WK (2007) A trait-based approach to community assembly: partitioning of species trait values into within-and amongcommunity components. Ecology Letters 10: 135-145.

Adler PB, Fajardo A, Kleinhesselink AR \& Kraft NJB (2013) Trait-based tests of coexistence mechanisms. Ecology Letters 16: 1294-1306.

Baker TR, Swaine MD \& Burslem DFRP (2003) Variation in tropical forest growth rates: combined effects of functional group composition and resource availability. Perspectives in Plant Ecology, Evolution and Systematics 6: 21-36.

Carreño-Rocabado G, Peña-Claros M, Bongers F, Alarcón A, Licona JC \& Poorter L (2012) Effects of disturbance intensity on species and functional diversity in a tropical forest. Journal of Ecology 100: 1453-1463.

Chave J (2006) Measuring wood density for tropical forest trees a field manual. Sixth Framework Programme, p. 6. Disponível em <http://www. rainfor.net/upload/ManualsEnglish/wood_density_ english\%5B1\%5D.pdf>. Acesso em 20 maio 2015.

Chave J, Coomes D, Jansen S, Lewis SL, Swenson NG \& Zanne AE (2009) Towards a worldwide wood economics spectrum. Ecology Letters 12: 351-366.

Cornwell WK \& Ackerly DD (2009) Community assembly and shifts in plant trait distributions across an environmental gradient in coastal California. Ecological Monographs 79: 109-126.

Díaz S \& Cabido M (2001) Vive la différence: plant functional diversity matters to ecosystem processes. Trends in Ecology and Evolution 16: 646-655.
Evans JR \& Poorter H (2001) Photosynthetic acclimation of plants to growth irradiance: the relative importance of specific leaf area and nitrogen partitioning in maximizing carbon gain. Plant, Cell and Environment 24: 755-767.

Formento S, Schorn LA \& Ramos RAB (2004) Dinâmica estrutural arbórea de uma Floresta Ombrófila Mista em Campo Belo do Sul, SC. Cerne 10: 196-212.

IBGE (2012) Manual técnico da vegetação brasileira. Fundação Instituto Brasileiro de Geografia e Estatística, Rio de Janeiro. 271p.

Jackson DA (1993) Stopping rules in principal components analysis: a comparison of heuristical and statistical approaches. Ecology 74: 2204-2214.

Laliberté E \& Legendre P (2010) A distance-based framework for measuring functional diversity from multiple traits. Ecology 91: 299-305.

Laliberté E \& Shipley B (2011) FD: measuring functional diversity from multiple traits, and other tools for functional ecology. R package version 1.0-11. Disponível em <https://cran.r-project.org/web/ packages/FD/citation.html>. Acesso em 30 julho 2016.

Leyser G, Zanin EM, Budke JC, Mélo MA \& HenkeOliveira C (2012) Regeneração de espécies arbóreas e relações com o componente adulto em uma floresta estacional no vale do Rio Uruguai, Brasil. Acta Botanica Brasilica 26: 74-83.

Losos JB \& Ricklefs RE (2009) Adaptation and diversification on islands. Nature 457: 830-836.

McGill BJ, Enquist BJ, Weiher E \& Westoby M (2006) Rebuilding community ecology from functional traits. Trends in Ecology and Evolution 21: 178-185.

Negrini M, Aguiar MD, Vieira CT, Silva AC \& Higuchi P (2012) Dispersão, distribuição espacial e estratificação vertical da comunidade arbórea em um fragmento florestal no Planalto Catarinense. Revista Árvore 36: 919-930.

Oksanen J, Blanchet FG, Kindt R, Legendre P, Minchin PR, O'Hara RB, Simpson GL, Solymos P, Stevens HH \& Wagner H (2016) Vegan: community ecology package. R package version 1: 8-8. Disponível em $<$ https://cran.r-project.org/web/packages/vegan/ index.html $>$. Acesso em 30 julho 2016.

Oliveira Filho AT, Vilela EA, Carvalho DA \& Gavilanes ML (1994) Effects of soils and topography on the distribution of tree species in a tropical riverine forest in south-eastern Brazil. Journal of Tropical Ecology 10: 483-508.

Oliveira Filho AT, Curi N, Vilela EA \& Carvalho DA (1998) Effects of canopy gaps, topography and soils on the distribution of woody species in a Central Brazilian deciduous dry forest. Biotropica 30: 362-375.

Pérez-Harguindeguy N, Díaz S, Garnier E, Lavorel S, Poorter H, Jaureguiberry P, Bret-Harte MS, Cornwell WK, Craine JM, Gurvich DE, Urcelay C, Veneklaas EJ, Reich PB, Poorter L, Wright IJ, 
Ray P, Enrico L, Pausas JG, De Vos AC, Buchmann N, Funes G, Quétier F, Hodgson JG, Thompson K, Morgan HD, Ter Steege H, Van der Heijden MGA, Sack L, Blonder B, Poschlod P, Vaieretti MV, Conti G, Staver AC, Aquino S \& Cornelissen JHC (2013) New handbook for standardised measurement of plant functional traits worldwide. Australian Journal of Botany 61: 167-234.

Poorter L, Bongers F, Sterck FJ \& Wöll H (2005) Beyond the regeneration phase: differentiation of heightlight trajectories among tropical tree species. Journal of Ecology 93: 256-267.

R Core Team (2016) R: a language and environment for statistical computing. R Foundation for Statistical Computing. Disponível em $<$ http://www.R-project. org >. Acesso em 15 abril 2016.

Reich PB (2014) The world-wide 'fast-slow' plant economics spectrum: a traits manifesto. Journal of Ecology 102: 275-301.

Rosado BHP, Joly CA, Burgess SSO, Oliveira RS \& Aidar MP (2016) Changes in plant functional traits and water use in Atlantic rainforest: evidence of conservative water use in spatio-temporal scales. Trees 30: 47-61.

Silva AC, Higuchi P, Aguiar MD, Negrini M, Fert Neto J \& Hess AF (2012) Relações florísticas e fitossociologia de uma Floresta Ombrófila Mista Montana Secundária em Lages, Santa Catarina. Ciência Florestal 22: 193-206.

Silvertown J, Dodd ME, Gowing DJG \& Mountford JO (1999) Hydrologically defined niches reveal a basis for species richness in plant communities. Nature 400: 61-63.

Souza K, Souza CC, Rosa MG, Cruz AP, Lima CL, Silva JO, Lazzarin LC, Loebens R, Dias RAR,
Silva AC, Higuchi P \& Schimalski MB (2015) Estrutura e estratégias de dispersão do componente arbóreo de uma floresta subtropical ao longo de uma topossequência no Alto-Uruguai. Scientia forestalis 43: 321-332.

Swaine MD \& Whitmore TC (1988) One the definition of ecological species groups in tropical rain forests. Vegetation 75: 81-86.

Thomas SC \& Winner WE (2002) Photosynthetic differences between saplings and adult trees: an integration of field results by meta-analysis. Tree physiology 22: 117-127.

Valencia R, Foster RB, Villa G, Condit R, Svenning JC, Hernández C, Romoleroux K, Losos E, Magard E \& Balslev H (2004) Tree species distributions and local habitat variation in the Amazon: large forest plot in eastern Ecuador. Journal of Ecology 92: 214-229.

Van der Pijl L (1982) Principles of dispersal in higher plants. Springer-Verlag, Berlin. 162p.

Violle C, Navas ML, Vile D, Kazakou E, Fortunel C, Hummel I \& Garnier E (2007) Let the concept of trait be functional!. Oikos 116: 882-892.

Violle C \& Jiang L (2009) Towards a trait-based quantification of species niche. Journal of Plant Ecology 2: 87-93.

Weiher E, Van den Werf A, Thompson K, Roderick M, Garnier E \& Eriksson O (1999) Challenging Theophrastus: a common core list of plant traits for functional ecology. Journal of Vegetation Science 10: 609-620.

Yang J, Ci X, Lu M, Zhang G, Cao M, Li J \& Lin L (2014) Functional traits of tree species with phylogenetic signal co-vary with environmental niches in two large forest dynamics plots. Journal of Plant Ecology 7: 115-125. 